\title{
A Hybrid Mean Value Involving Dedekind Sums and the General Exponential Sums
}

\author{
Jianghua $\mathrm{Li}^{1}$ and Tingting Wang ${ }^{2}$ \\ ${ }^{1}$ College of Science, Xian University of Technology, Xian, Shaanxi 710048, China \\ ${ }^{2}$ College of Science, Northwest A\&F University, Yangling District, Shaanxi 712100, China \\ Correspondence should be addressed to Tingting Wang; tingtingwang126@126.com
}

Received 13 May 2014; Revised 9 July 2014; Accepted 20 July 2014; Published 7 August 2014

Academic Editor: Reza Ansari

Copyright (c) $2014 \mathrm{~J}$. Li and T. Wang. This is an open access article distributed under the Creative Commons Attribution License, which permits unrestricted use, distribution, and reproduction in any medium, provided the original work is properly cited.

\begin{abstract}
The main purpose of this paper is using the analytic method, A. Weil's classical work for the upper bound estimate of the general exponential sums, and the properties of Gauss sums to study the hybrid mean value problem involving Dedekind sums and the general exponential sums and give a sharp asymptotic formula for it.
\end{abstract}

\section{Introduction}

For a positive integer $k$ and an arbitrary integer $h$, the classical Dedekind sums $S(h, k)$ are defined by

$$
S(h, k)=\sum_{a=1}^{k}\left(\left(\frac{a}{k}\right)\right)\left(\left(\frac{a h}{k}\right)\right)
$$

where

$$
((x))= \begin{cases}x-[x]-\frac{1}{2}, & \text { if } x \text { is not an integer } \\ 0, & \text { if } x \text { is an integer. }\end{cases}
$$

The various properties of $S(h, k)$ were investigated by many authors; see [1-5]. For example, Conrey et al. [3] studied the mean value distribution of $S(h, k)$ and proved the asymptotic formula

$$
\begin{aligned}
\sum_{h=1}^{\prime}|S(h, k)|^{2 m}= & f_{m}(k)\left(\frac{k}{12}\right)^{2 m} \\
& +O\left(\left(k^{9 / 5}+k^{2 m-1+(1 /(m+1))}\right) \cdot \ln ^{3} k\right)
\end{aligned}
$$

where $\sum_{h}^{\prime}$ denotes the summation over all $h$ such that $(k, h)=$ 1 , and

$$
\sum_{m=1}^{\infty} \frac{f_{m}(n)}{n^{s}}=2 \cdot \frac{\zeta^{2}(2 m)}{\zeta(4 m)} \cdot \frac{\zeta(s+4 m-1)}{\zeta^{2}(s+2 m)} \cdot \zeta(s) .
$$

Zhang [6] established a close contact between $S(h, q)$ and the mean square value of Dirichlet $L$-functions (see Lemma 4). Maybe the most important property of $S(h, q)$ is its reciprocity theorem (see [2]). That is, for all positive integers $h$ and $q$ with $(h, q)=1$, we have the identity

$$
S(h, q)+S(q, h)=\frac{h^{2}+q^{2}+1}{12 h q}-\frac{1}{4} .
$$

On the other hand, Liu and Zhang [5] studied the hybrid mean value problem involving $S(h, k)$ and $R_{q}(c)$ and proved the following conclusion.

Let $q>2$ be a square-full number; then

$$
\sum_{h=1}^{q} S(h, q) R_{q}(h+1)=-\frac{1}{3} \cdot q \cdot \phi^{2}(q) \cdot \prod_{p \mid q}\left(1+\frac{1}{p}\right)
$$


where $R_{q}(c)$ is the Ramanujan sum, defined as (see Theorem 8.6 of [7])

$$
R_{q}(c)=\sum_{\substack{k=1 \\(k, q)=1}}^{q} e^{2 \pi i k c / q}=\sum_{d \mid(c, q)} d \mu\left(\frac{q}{d}\right),
$$

and $\mu(n)$ is the famous Möbius function.

Weil [8] studied the upper bound estimate of the general exponential sums

$$
E(f(x), p)=\sum_{a=1}^{p} e\left(\frac{f(a)}{p}\right)
$$

and proved the estimate (see Corollary $2 \mathrm{~F}$ of [9, page 45])

$$
E(f(x), p) \ll_{k} \sqrt{p},
$$

where $e(x)=e^{2 \pi i x}, p$ is an odd prime, $\ll_{k}$ denotes the big$O$ constant which depends only on $k$, and $f(x)=a_{k} x^{k}+$ $a_{k-1} x^{k-1}+\cdots+a_{1} x+a_{0}$ is $k$ th integral coefficients polynomial with $\left(a_{k}, p\right)=1$.

In fact, the estimate (9) is the best one, since if $f(x)=x^{2}$, then from Gauss famous work (see [7, page 195]) we have

$$
\left|\sum_{a=1}^{p} e\left(\frac{a^{2}}{p}\right)\right|=\sqrt{p} .
$$

The content and form of this paper are different from the references $[3,6]$. Conrey et al. [3] studied the general $2 k$ th power mean of Dedekind sums and obtained an asymptotic formula. Zhang [6] only obtained a relationship between $S(h, q)$ and the mean square value of Dirichlet $L$-functions. Our work is using Zhang's result, Weil's classical work for the upper bound estimate of the general exponential sums, and the properties of Gauss sums to study the hybrid mean value problem involving Dedekind sums and the general exponential sums $E(f(x), p)$ and give a sharper asymptotic formula for it.

\section{Main Theorems}

In this paper, we will obtain the following two results.

Theorem 1. Let $p$ be an odd prime and let $k$ be any fixed positive integer. Suppose that $f(x)=a_{k} x^{k}+a_{k-1} x^{k-1}+\cdots+a_{1} x+$ $a_{0}$ is a polynomial with integral coefficients having $0<k<p$ and $\left(a_{1}, p\right)=1$. Then we have the asymptotic formula

$$
\begin{gathered}
\sum_{m=1}^{p-1} \sum_{n=1}^{p-1} E(m x+f(x), p) \cdot E(n x-f(x), p) \cdot S(m \cdot \bar{n}, p) \\
=\frac{1}{12} \cdot p^{3}+O\left(p^{5 / 2} \cdot \ln p\right)
\end{gathered}
$$

where $\bar{n}$ denotes the solution of the congruence equation $n x \equiv$ $1 \bmod p$.
For the classical Kloosterman sums, we can also obtain a similar conclusion. That is, we have the following.

Theorem 2. Let $p$ be an odd prime; then we have the asymptotic formula

$$
\begin{gathered}
\sum_{m=1}^{p-1} \sum_{n=1}^{p-1} K(m+1, p) \cdot K(n+1, p) \cdot S(m \cdot \bar{n}, p) \\
=\frac{1}{12} \cdot p^{3}+O\left(p^{5 / 2} \cdot \ln p\right),
\end{gathered}
$$

where $K(m, p)=\sum_{a=1}^{p-1} e((m a+\bar{a}) / p)$ denotes the classical Kloosterman sums.

For general integer $q>3$, whether there exists a similar asymptotic formula as in Theorem 1 (or Theorem 2 ) is an open problem.

\section{Lemmas and Proofs of the Theorems}

In order to complete the proof of our theorems, we need the following several simple lemmas. Hereinafter, we will use many definitions and properties of Gauss sums, Kloosterman sums, and character sums, all of which can be found in $[7,10-$ 13], so they will not be repeated here. First we have the following.

Lemma 3. Let $p$ be an odd prime and let $\chi$ be the Dirichlet character $\bmod p$. Then one has the estimate

$$
\left.\sum_{r=1}^{p-1}\left|\sum_{\substack{\chi \bmod p \\ \chi(-1)=-1}} \chi(r)\right| L(1, \chi)\right|^{2} \mid=O(p \cdot \ln p),
$$

where $\sum_{\chi \bmod p_{\chi(-1)=-1}}$ denotes the summation over all odd characters $\chi \bmod p$.

Proof. From the method of proving Lemma 5 in [14] we may immediately deduce this estimate.

Lemma 4. Let $q>2$ be an integer; then for any integer $a$ with $(a, q)=1$, one has the identity

$$
S(a, q)=\frac{1}{\pi^{2} q} \sum_{d \mid q} \frac{d^{2}}{\phi(d)} \sum_{\substack{\chi \bmod d \\ \chi(-1)=-1}} \chi(a)|L(1, \chi)|^{2},
$$

where $L(1, \chi)$ denotes the Dirichlet $L$-function corresponding to character $\chi \bmod d$. 
Proof. See Lemma 2 of [6].

Lemma 5. Let $p$ be an odd prime; then one has the asymptotic formula

$$
\begin{gathered}
\sum_{\substack{\chi \bmod p \\
\chi(-1)=-1}}\left|\sum_{a=1}^{p-1} \chi(a) \cdot e\left(\frac{a+\bar{a}}{p}\right)\right|^{2} \cdot|L(1, \chi)|^{2} \\
=\frac{\pi^{2}}{12} \cdot p^{2}+O\left(p^{3 / 2} \cdot \ln p\right) .
\end{gathered}
$$

Proof. See the theorem and corollary of [14].

Proof of Theorem 1. From Lemma 4 with $q=p$, an odd prime, we have

$$
\begin{aligned}
& \sum_{\substack{\chi \bmod p \\
\chi(-1)=-1}}|L(1, \chi)|^{2} \\
&= \frac{\pi^{2}(p-1)}{p} \sum_{a=1}^{p-1}\left(\frac{a}{p}-\frac{1}{2}\right)^{2}=\frac{\pi^{2}}{12} \cdot \frac{(p-1)^{2}(p-2)}{p^{2}}, \\
& \sum_{m=1}^{p-1} \sum_{n=1}^{p-1} E(m x+f(x), p) \cdot E(n x-f(x), p) \cdot S(m \cdot \bar{n}, p) \\
&=\frac{p}{\pi^{2}(p-1)} \sum_{\substack{\chi \bmod p \\
\chi(-1)=-1}}^{p-1} \sum_{m=1} \chi(m) E(m x+f(x), p) \\
& \times \sum_{n=1}^{p-1} \bar{\chi}(n) E(n x-f(x), p)|L(1, \chi)|^{2} \\
&= \frac{p}{\pi^{2}(p-1)} \sum_{\substack{\chi \bmod p \\
\chi(-1)=-1}}\left|\sum_{m=1}^{p-1} \chi(m) E(f(x)+m x, p)\right|^{2} \\
& \cdot|L(1, \chi)|^{2} .
\end{aligned}
$$

From the definition of $E(f(x), p)$ and the properties of Gauss sum, we have

$$
\begin{aligned}
\sum_{m=1}^{p-1} \chi & (m) E(f(x)+m x, p) \\
& =\sum_{a=1}^{p-1} \sum_{m=1}^{p-1} \chi(m) \cdot e\left(\frac{f(a)+m a}{p}\right) \\
& =\sum_{a=1}^{p-1} e\left(\frac{f(a)}{p}\right) \sum_{m=1}^{p-1} \chi(m) \cdot e\left(\frac{m a}{p}\right) \\
& =\tau(\chi) \cdot \sum_{a=1}^{p-1} \bar{\chi}(a) \cdot e\left(\frac{f(a)}{p}\right) .
\end{aligned}
$$

Note that for any nonprincipal character $\chi \bmod p$, we have $|\tau(\chi)|=\sqrt{p}$. For any integer $2 \leq a \leq p-1$, since $\left(a_{1}(a-\right.$ $1), p)=1$, the polynomial $f(a x)-f(x)$ satisfying $p \nmid(f(a x)-$ $f(x)$ ). Applying (9), we have the estimate

$$
\left|\sum_{b=1}^{p-1} e\left(\frac{f(a b)-f(b)}{p}\right)\right| \ll \sqrt{p} .
$$

Now combining (16), (17), (18), (19), and Lemma 3, we have the asymptotic formula

$$
\begin{aligned}
& \sum_{m=1}^{p-1} \sum_{n=1}^{p-1} E(m x+f(x), p) \cdot E(n x-f(x), p) \cdot S(m \cdot \bar{n}, p) \\
& =\frac{p^{2}}{\pi^{2}(p-1)} \sum_{\substack{\chi \bmod p \\
\chi(-1)=-1}}\left|\sum_{a=1}^{p-1} \bar{\chi}(a) \cdot e\left(\frac{f(a)}{p}\right)\right|^{2} \cdot|L(1, \chi)|^{2} \\
& =\frac{p^{2}}{\pi^{2}(p-1)} \sum_{\substack{\chi \bmod p \\
\chi(-1)=-1}} \sum_{a=1}^{p-1} \sum_{b=1}^{p-1} \bar{\chi}(a) \chi(b) \\
& \cdot e\left(\frac{f(a)-f(b)}{p}\right) \cdot|L(1, \chi)|^{2} \\
& =\frac{p^{2}}{\pi^{2}(p-1)} \sum_{\substack{\chi \bmod p \\
\chi(-1)=-1}} \sum_{a=1}^{p-1} \bar{\chi}(a) \sum_{b=1}^{p-1} e\left(\frac{f(a b)-f(b)}{p}\right) \\
& \cdot|L(1, \chi)|^{2} \\
& =\frac{p^{2}}{\pi^{2}(p-1)} \sum_{a=2}^{p-1}\left(\sum_{b=1}^{p-1} e\left(\frac{f(a b)-f(b)}{p}\right)\right) \\
& \cdot\left(\sum_{\substack{\chi \bmod p \\
\chi(-1)=-1}} \bar{\chi}(a)|L(1, \chi)|^{2}\right) \\
& +\frac{p^{2}}{\pi^{2}} \sum_{\substack{\chi \bmod p \\
\chi(-1)=-1}}|L(1, \chi)|^{2} \\
& =\frac{p^{2}}{\pi^{2}} \cdot \frac{\pi^{2}}{12} \cdot \frac{(p-1)^{2}(p-2)}{p^{2}} \\
& +O\left(\left.p \sum_{a=2}^{p-1} \sqrt{p} \cdot\left|\sum_{\substack{\chi \bmod p \\
\chi(-1)=-1}} \bar{\chi}(a)\right| L(1, \chi)\right|^{2} \mid\right) \\
& =\frac{p^{2}}{\pi^{2}} \cdot \frac{\pi^{2}}{12} \cdot \frac{(p-1)^{2}(p-2)}{p^{2}}
\end{aligned}
$$




$$
\begin{aligned}
& +O\left(\left.p^{3 / 2} \cdot \sum_{a=1}^{p-1}\left|\sum_{\substack{\chi \bmod p \\
\chi(-1)=-1}} \bar{\chi}(a)\right| L(1, \chi)\right|^{2} \mid\right) \\
= & \frac{1}{12} \cdot p^{3}+O\left(p^{5 / 2} \cdot \ln p\right) .
\end{aligned}
$$

This proves Theorem 1.

Proof of Theorem 2. Note that if $\chi(-1)=-1$, then $\tau(\bar{\chi})=$ $-\overline{\tau(\chi)}$. Consider

$$
\begin{aligned}
& \sum_{m=1}^{p-1} \chi(m) K(m+1, p) \\
& =\sum_{a=1}^{p-1} \sum_{m=1}^{p-1} \chi(m) \cdot e\left(\frac{(m+1) a+\bar{a}}{p}\right) \\
& =\tau(\chi) \sum_{a=1}^{p-1} \bar{\chi}(a) \cdot e\left(\frac{a+\bar{a}}{p}\right) \\
& \sum_{a=1}^{p-1} \bar{\chi}(a) \cdot e\left(\frac{a+\bar{a}}{p}\right)=\bar{\chi}(-1) \cdot \sum_{a=1}^{p-1} \chi(a) \cdot e\left(\frac{a+\bar{a}}{p}\right) .
\end{aligned}
$$

From (16), the properties of Gauss sums, and Lemma 5, we have

$$
\begin{aligned}
& \sum_{m=1}^{p-1} \sum_{n=1}^{p-1} K(m+1, p) \cdot K(n+1, p) \cdot S(m \cdot \bar{n}, p) \\
& =\frac{p}{\pi^{2}(p-1)} \sum_{\substack{\chi \bmod p \\
\chi(-1)=-1}} \sum_{m=1}^{p-1} \chi(m) K(m+1, p) \\
& \cdot \sum_{n=1}^{p-1} \bar{\chi}(n) K(m+1, p)|L(1, \chi)|^{2} \\
& =\frac{p}{\pi^{2}(p-1)} \sum_{\substack{\chi \bmod p \\
\chi(-1)=-1}}|\tau(\chi)|^{2} \\
& \cdot\left|\sum_{a=1}^{p-1} \bar{\chi}(a) \cdot e\left(\frac{a+\bar{a}}{p}\right)\right|^{2} \cdot|L(1, \chi)|^{2} \\
& =\frac{p^{2}}{\pi^{2}(p-1)} \sum_{\substack{\chi \bmod p \\
\chi(-1)=-1}}\left|\sum_{a=1}^{p-1} \chi(a) \cdot e\left(\frac{a+\bar{a}}{p}\right)\right|^{2} \\
& \cdot|L(1, \chi)|^{2} \\
& =\frac{1}{12} \cdot p^{3}+O\left(p^{5 / 2} \cdot \ln p\right) \text {. }
\end{aligned}
$$

This completes the proof of Theorem 2.

\section{Conflict of Interests}

The authors declare that they have no conflict of interests.

\section{Acknowledgments}

The authors would like to thank the referees for their very helpful and detailed comments, which have significantly improved the presentation of this paper. This work is supported by the Specific Fundation of Xian University of Technology (no. 2013TS011) and the Specific Fundation for Talents of Northwest A\&F University.

\section{References}

[1] T. M. Apostol, Modular Functions and Dirichlet Series in Number Theory, Springer, New York, NY. USA, 1976.

[2] L. Carlitz, “The reciprocity theorem of Dedekind Sums," Pacific Journal of Mathematics, vol. 3, pp. 513-522, 1953.

[3] J. B. Conrey, E. Fransen, R. Klein, and C. Scott, "Mean values of Dedekind sums," Journal of Number Theory, vol. 56, no. 2, pp. 214-226, 1996.

[4] C. Jia, "On the mean value of Dedekind sums," Journal of Number Theory, vol. 87, no. 2, pp. 173-188, 2001.

[5] H. Y. Liu and W. P. Zhang, "Some identities involving certain Hardy sums and Ramanujan sum," Acta Mathematica Sinica, vol. 21, no. 1, pp. 109-116, 2005.

[6] W. Zhang, "On the mean values of Dedekind sums," Journal de Theorie des Nombres de Bordeaux, vol. 8, no. 2, pp. 429-442, 1996.

[7] T. M. Apostol, Introduction to Analytic Number Theory, Springer, New York, NY, USA, 1976.

[8] A. Weil, "On some exponential sums," Proceedings of the National Academy of Sciences of the United States of America, vol. 34, pp. 204-207, 1948.

[9] W. M. Schmidt, Equations Over Finite Fields-An Elementary Approach, Springer, New York, NY, USA, 1976.

[10] S. Chowla, On Kloostermann's Sum, vol. 40 of Kongelige Norske Videnskabers Selskab; Forhandlinger, 1967.

[11] J.-M. Deshouillers and H. Iwaniec, "Kloosterman sums and Fourier coefficients of cusp forms," Inventiones Mathematicae, vol. 70, no. 2, pp. 219-288, 1982.

[12] A. V. Malyshev, "A generalization of Kloostermann sums and their estimates," Vestnik Leningrad University, vol. 15, pp. 59-75, 1960 (Russian).

[13] H. Salié, "Über die Kloostermanschen Summen S(u, v; q)," Mathematische Zeitschrift, vol. 34, no. 1, pp. 91-109, 1932.

[14] W. Zhang, Y. Yi, and X. He, "On the $2 k$-th power mean of Dirichlet $L$-functions with the weight of general Kloosterman sums," Journal of Number Theory, vol. 84, no. 2, pp. 199-213, 2000. 


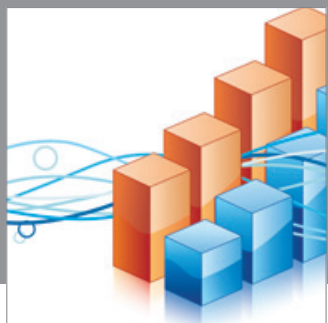

Advances in

Operations Research

mansans

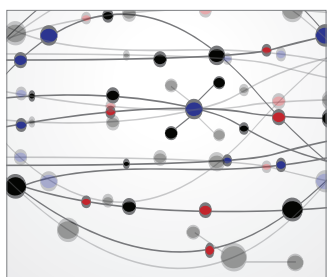

The Scientific World Journal
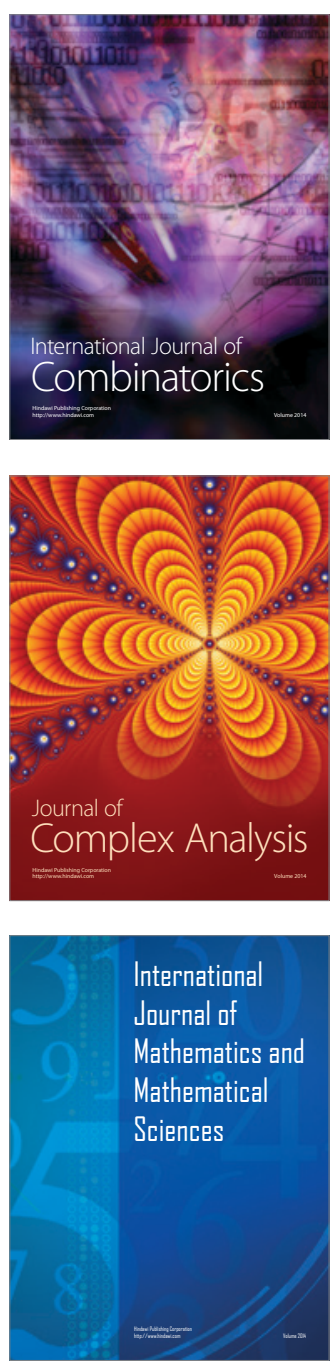
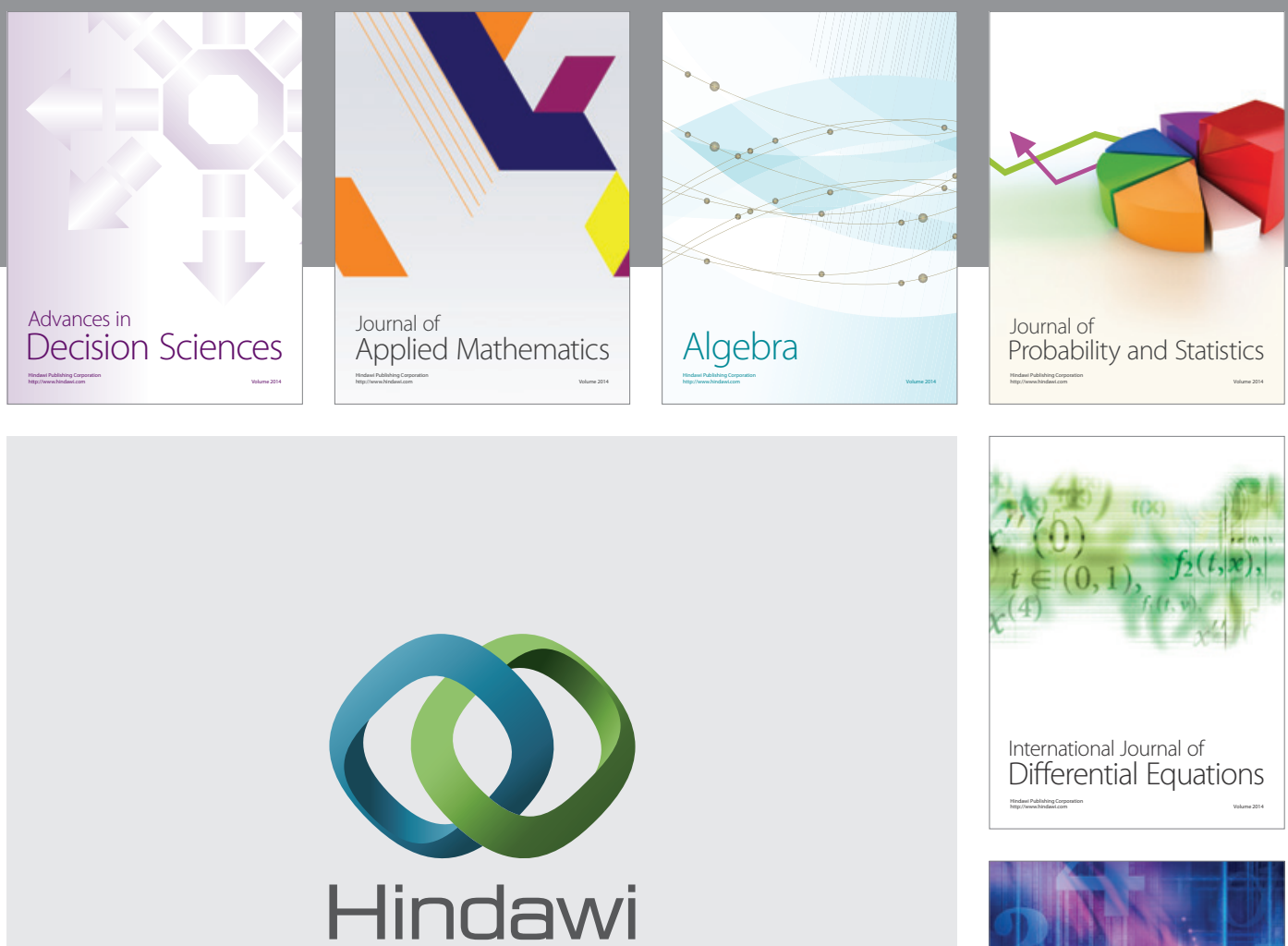

Submit your manuscripts at http://www.hindawi.com
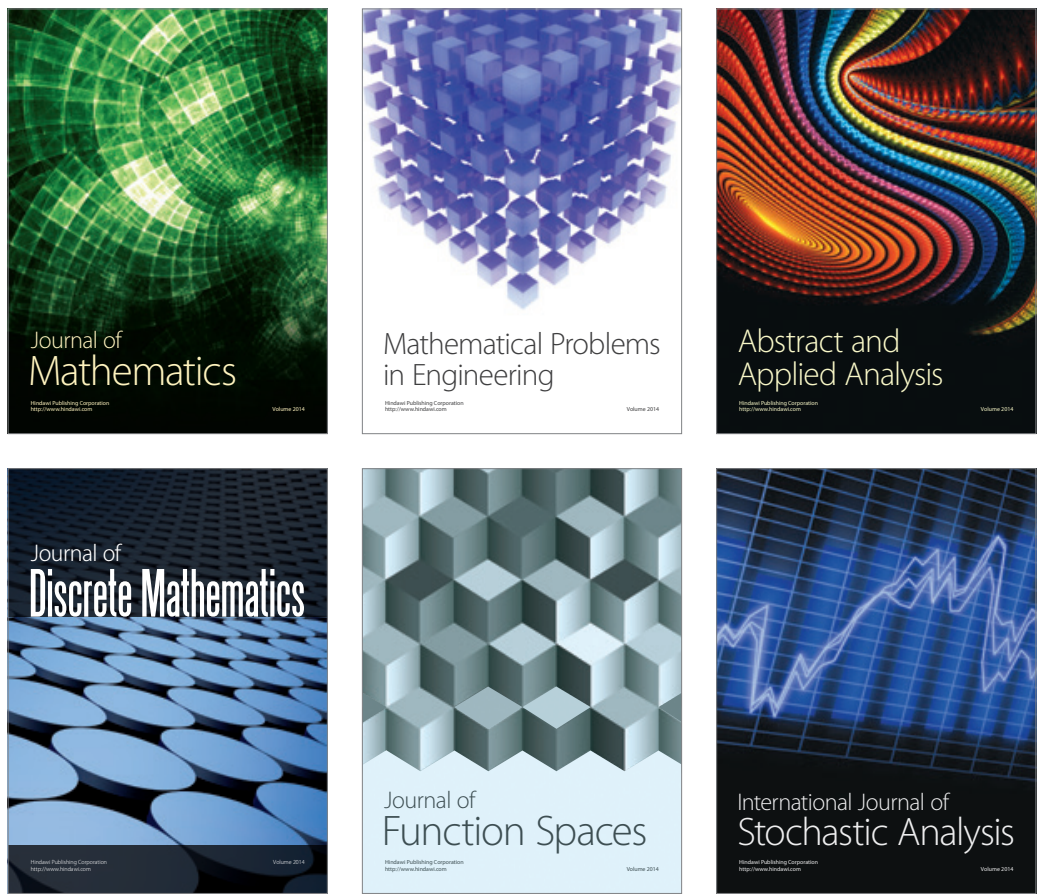

Journal of

Function Spaces

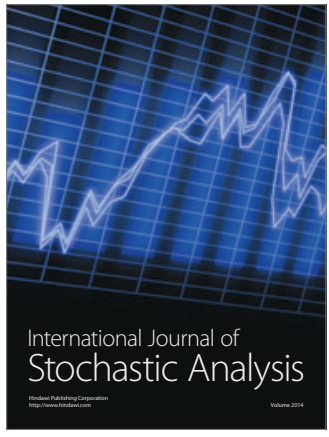

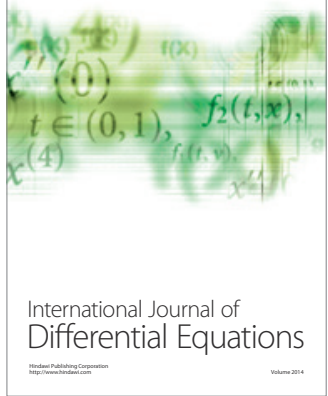
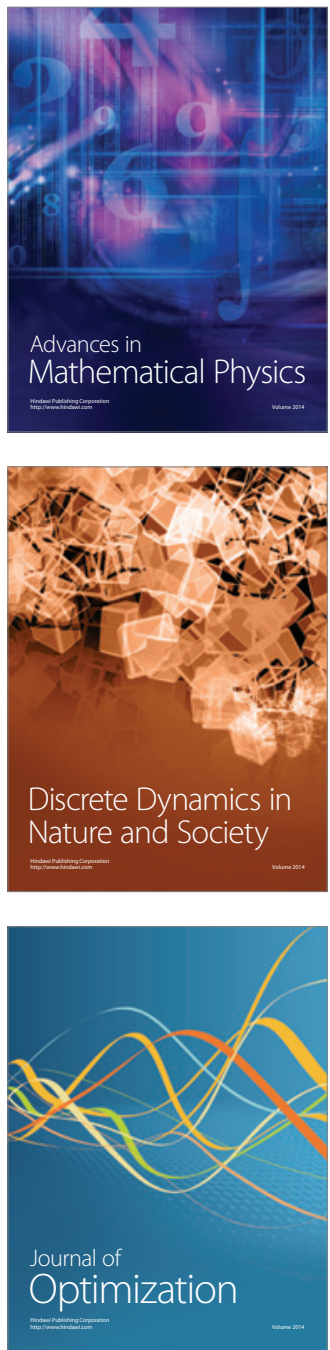